\title{
Brandrodungsfeldbau in Liberia (Shifting Cultivation, Wald-Feld-Wechselwirtschaft, SWA S. 91)
}

\section{Vorbemerkungen}

«Der Mensch im tropischen Regenwald», «Urwaldindianer» oder "Hackbauern in Afrika» sind beliebte Themen, um die Beziehungen zwischen Mensch und Raum darzustellen. Die Beschränkung auf Extremräume und naturverbundene traditionelle Lebensformen und Wertsysteme erleichtert die Einsicht in Mensch-Natur-Beziehungen. In diesem Sinn ist das Raumbeispiel Brandrodungsfeldbau in Liberia geeignet zum Einstieg ins Interpretieren thematischer Karten. Eine vertiefte Auseinandersetzung mit dem Thema setzt allerdings einige Informationen voraus.

\section{Bedeutung des Brandrodungsfeldbaus}

Beim Brandrodungsfeldbau (Shifting Cultivation) handelt es sich um das in den feuchten Tropen noch weit verbreitete, älteste und wenig produktive Landnutzungssystem der einheimischen Bevölkerung. Im Gebiet des Regenwaldes und der Feuchtsavanne, d. h. auf rund 30 Mio. km² beziehungsweise auf $30 \%$ der agrarisch nutzungsfähigen Oberfläche der Erde, betreiben heute mehr als 250 Millionen Hackbauern irgendeine Variante des Brandrodungsfeldbaus. Eines der auffallendsten Merkmale dieser Zone ist die relativ geringe Bevölkerungsdichte, für die vor allem das mangelhafte Agrarpotential verantwortlich gemacht wird. Tatsächlich sind große Teile sowohl der lateinamerikanischen wie auch afrikanischen und südostasiatischen Regenwaldzone noch immer dünn besiedelt. In Südostasien, z. B. auf den Außeninseln Indonesiens, auf Papua-Neuguinea, bei den Indianern im Amazonasgebiet und in den dauerfeuchten Tropen Afrikas ist Shifting Cultivation nach wie vor das flächenmäBig vorherrschende Bodennutzungssystem. Brandrodungsfeldbau ist nicht nur ein Agrarsystem, sondern vielmehr eine Kulturform der Regenwaldvölker (Stammesvölker), die seit Jahrhunderten mit dem Ökosystem «Tropischer Regenwald" umzugehen wissen, nun aber infolge des kulturellen Umbruchs und angesichts des zunehmenden Bevölkerungsdrucks vor eine entscheidende Herausforderung gestellt werden.

\section{Das Agrarsystem Shifting Cultivation}

Weltweit wird Shifing Cultivation in vielen Varianten betrieben; Anbaufolgen, Anbauprodukte, aber auch Ar- beitsmethoden und Arbeitsorganisation sind regional unterschiedlich. Bei allen Formen handelt es sich aber um einen vergleichbaren Produktionsablauf, um Brandrodung, um Mischkulturen und um mehr oder weniger stark subsistenzorientierte Familienbetriebe mit niedrigem Lebensstandard und hoher ökologischer Angepaßtheit.

Beim Brandrodungsfeldbau, auch Wald-Feld-Wechselwirtschaft oder Landwechselwirtschaft genannt, werden auf der Suche nach fruchtbarem Land häufig jährlich neue Brandrodungen gemacht und die vorjährigen Pflanzungen verlassen. Deshalb zeigen die Luftbilder ein Mosaik unregelmäßig geformter, verteilter und getönter Waldparzellen. Einzelne Teile erweisen sich als frische Rodungen, andere mit niederem, jungem Busch oder Wald lassen auf einstige Rodungen schließen. Typisch ist also ein vorübergehender Anbau mit darauf folgender langer Brache zur Regenerierung des Waldes und des Bodens. Die ursprüngliche Variante des Wanderfeldbaus, bei welcher episodisch oder periodisch auch die Siedlung verlagert wird, ist nur bei äußerst geringer Bevölkerungsdichte mit unbegrenzter Flächenverfügbarkeit möglich und kommt nur noch in abgelegensten Gebieten vor.

Die am Raumbeispiel Zimgbo Town im Hinterland von Monrovia dargestellte Nutzungsfolge ist eine von vielen regional unterschiedlichen Formen der Shifting Cultivation. Da in Liberia seit Weihnachten 1989 Bürgerkrieg herrscht, sind etwa 700000 Flüchtlinge in die Nachbarländer geflohen. Dabei wurde die Bevölkerung vieler Dörfer in die Flucht getrieben, so daß heute viele Dörfer zerstört oder von andern Leuten bewohnt sind (1 Mio. Binnenflüchtlinge). Das Land liegt im Chaos und ist wohl um viele Jahre wirtschaftlich zurückgeworfen. Man wird folglich im Hinterland erst recht und noch lange Zeit auf das beschriebene Brandrodungssystem angewiesen sein. Vor dem Bürgerkrieg hatten in Liberia gegen 1,5 Mio. Bauern (70\% der Bevölkerung) Brandrodungsfeldbau betrieben.

\section{Kartenauswertung: Brandrodungsfeldbau in Liberia (Beispiel für die Orientierungsstufe)}

Beim Kartenauswerten hat sich folgendes Vorgehen bewährt, das in ähnlicher Weise auf alle thematischen Karten angewendet werden kann.

Peter Koch, Dr., Buggenacher 27, 6043 Adligenswil 


\section{Erster Schritt: Einlesen in die Karte (SWA S. 91)}

1. Thema erfassen: Kartentitel, Legendenaufbau überfliegen. Was fällt beim ersten Blick auf die Karte auf? Was möchte die Karte vermitteln? Lösung: Brandrodungsfeldbau in Liberia, Landnutzung.

2. Raum lokalisieren: Abgrenzung, Gradnetzangaben, Lageeinordnung des abgebildeten Raumes in der Übersichtskarte (SWA S. 92): Im Hinterland von Monrovia.

3. Maßstab bewußt zur Kenntnis nehmen. Flächengröße des Kartenausschnittes abschätzen: $M 1: 20000,3,5 \mathrm{~km}^{2}$.

4. Legende beachten, Erläuterungen lesen: Was ist dargestellt? Flächenfarben zur Darstellung der Pflanzungen, der Buschbrachen und der Waldstadien.

5. Zur Auswertung: Wie lautet die Aufgabe? Zum Beispiel: Beschreibe den Brandrodungsfeldbau von Zimgbo Town oder: Löse die folgenden Aufgaben.

\section{Zweiter Schritt: Karteninhalt beschreiben}

1. Erfassen der Basiskarte: Beschreibe das Relief und das Gewässernetz, die Lage des Dorfes und das Verkehrsnetz. Schätze die Einwohnerzahl (beachte, daß infolge Generalisierung etwa ein Viertel der kleineren Häuser nicht dargestellt ist).

2. Erfassen des Themas: Wie ist das Thema Brandrodungsfeldbau dargestellt?

a) Identifiziere die verschiedenen Farbflächen.

b) Beschreibe die Flurformen (Umrisse und Umgebung der Felder), schätze die Größe der Pflanzungen. Wo liegen die Reispflanzungen bezüglich Relief und Dorfzentrum?

c) Welches Hauptnahrungsmittel produzieren die Bauern von Zimgbo Town, welches Nahrungsmittel steht an zweiter Stelle, was können sie sonst noch ernten, vor allem in einem ganz schmalen Gürtel ums Dorf herum?

d) Zeige, wo die Bauern ihre Reisfelder im letzten und wo im vorletzten Jahr angelegt hatten. Was wächst jetzt dort? Was kann man daraus schließen?

e) Skizziere die Reispflanzungen auf Millimeter-Pauspapier. Schätze den Flächenanteil dieser Felder an der Gesamtfläche des Dorfes.

f) Wie lange wird dasselbe Feld genutzt, wie viele Jahre liegt es nachher brach?

\section{Dritter Schritt: Karteninhalt erklären (Beizug ergänzender Karten)}

\section{Erfassen der Ursachen, Folgerungen}

a) In welcher Klima- und Vegetationszone liegt Liberia (SWA S. 92/94)?

b) Welches Klimadiagramm (SWA S. 90) könnte ähnlich sein wie das Klima im Küstengebiet von Liberia (S. 90/103)? Beschreibe das Klima aufgrund der Klimakarten (SWA S. 90/103). c) Wie ist die Bevölkerungsdichte im Hinterland von Monrovia (SWA S. 96)?

d) Um welche Böden handelt es sich in Zimgbo Town (SWA S. 154)?

e) Wie wird diese Landwirtschaftsform von Zimgbo Town auch genannt? Siehe die Wirtschaftsformen S. 93 und die Landnutzung S. 162.1.

f) Erläutere nun Anbautechnik und Anbauzyklus (beachte auch den Titel der Karte!). Wovon hängt die Brachedauer $a b$ ?

g) Wie könnte der Landbesitz geregelt sein? Beachte zur Beantwortung die unregelmäßige Form der Felder, warum sind es nicht Äcker wie bei uns?

h) Betreibt man in diesem Dorf auch Viehhaltung? Die Ernährungskarte S.162.2 und die Karte S.163.2 Fleischproduktion und Verfügbarkeit an Eiweißen geben Auskunft.

\section{Zusammenfassung}

Was ist charakteristisch am Brandrodungsfeldbau? Welches könnten die Gründe für dieses Landnutzungssystem sein? Nenne Vor- und Nachteile. Vergleiche mit den Bauern in einem Schweizer Dorf bezüglich Flächenaufwandes, Arbeitseinsatzes und Ernteertrags.

Grenzen der Auswertbarkeit erkennen. Welche Informationen fehlen? Wie und woher könnten weitere Informationen beschafft werden?

\section{Bei den Hackbauern in Zimgbo Town (Liberia)}

\section{a) Das Dorf und seine Umwelt}

Zimgbo Town (Zumbata ist der Name auf älteren Karten) liegt $40 \mathrm{~km}$ (Luftlinie) nordöstlich von Monrovia und umfaßt knapp $7 \mathrm{~km}^{2}$ niedriges Hügelland. Die Atlaskarte zeigt nur die Hälfte des Dorfgebietes. Das Dorf liegt in einer Talung $53 \mathrm{~m}$ ü. M. Der Höhenunterschied zwischen Hügelrücken und Talungen beträgt 40 bis $70 \mathrm{~m}$.

Das äquatoriale Regenwaldklima ist monsunal beeinflußt und hat deshalb eine kurze Trockenzeit von Dezember bis Februar. Weitere Angaben entnehme man den Klimakarten im Atlas (S. 90 u. 103). Allerdings regnet es mit $325 \mathrm{~cm}$ jährlich erheblich weniger als in Monrovia. Die relative Feuchtigkeit beträgt fast das ganze Jahr $80-100 \%$.

Ursprünglich bedeckte der feuchte immergrüne Regenwald die ganze Region. Seit 100 Jahren ist diese Gegend immer dichter besiedelt worden, so daß infolge Brandrodungsfeldbaus nur noch degenerierter Busch- oder Sekundärwald vorhanden ist. Nur vereinzelt sind noch alte Baumriesen übriggeblieben. Typisches Zeichen für Sekundärwuchs sind die vielen Ölpalmen in den Wäldern. Dorf und Landschaft zeigen die für viele liberianischen Dörfer charakteristischen Merkmale: das ohne festen Grundriß in einer gerundeten Lichtung des Regenwaldes errichtete Haufendorf und das durch den Brandrodungsfeldbau geprägte Landschaftsbild. Frische Rodungen, Pflanzungen, Busch- und Sekundärwald aller Alters- 


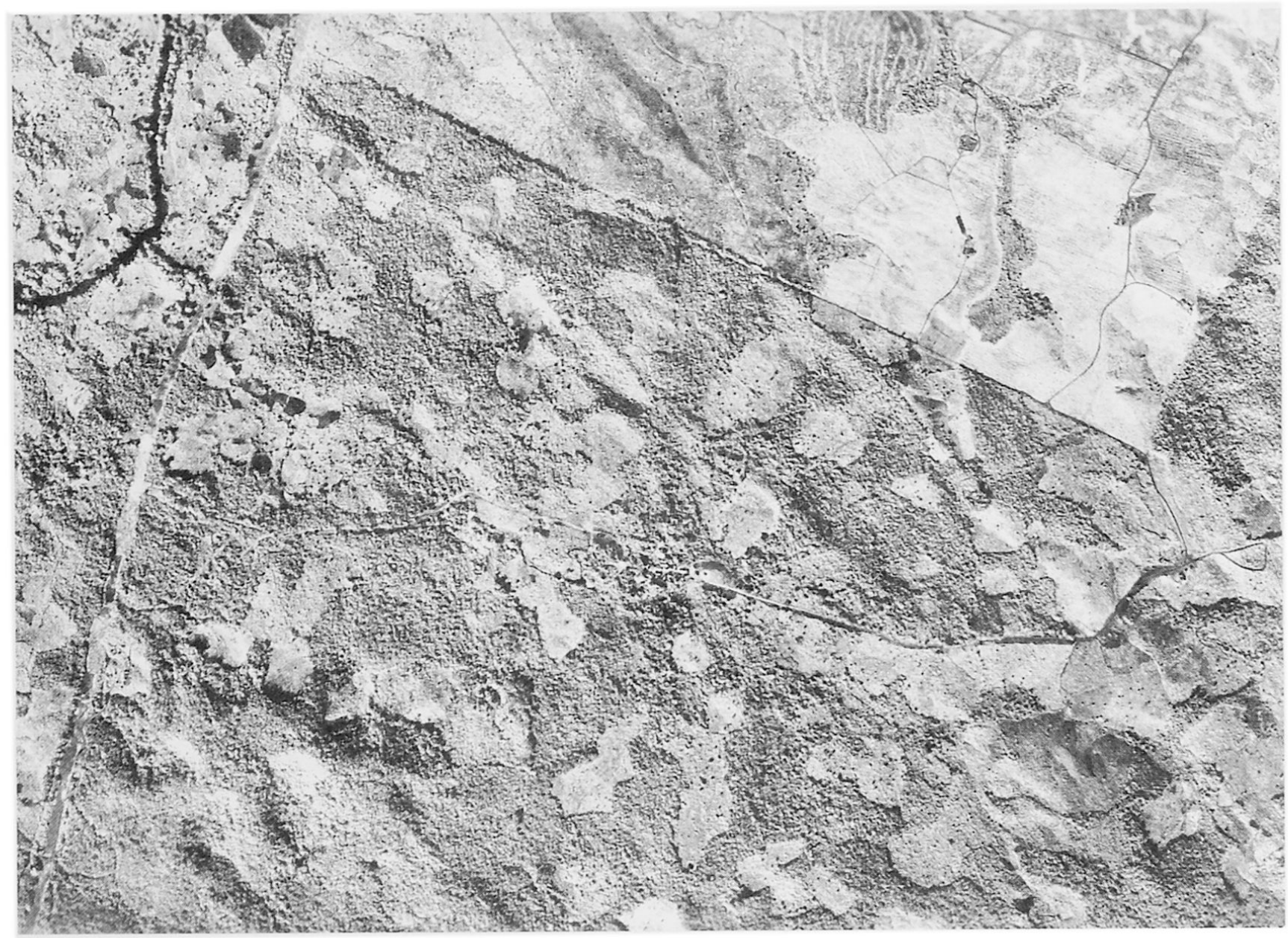

Abb.1 Grundlage zur Vegetations- und Landnutzungskartierung waren Luftbilder verschiedener Jahre. Das Luftbild Zimgbo Town (Infrarotaufnahme im Maßstab 1:25 500, Flughöhe um 3900 m ü. G.) diente zur Herstellung der Atlaskarte.

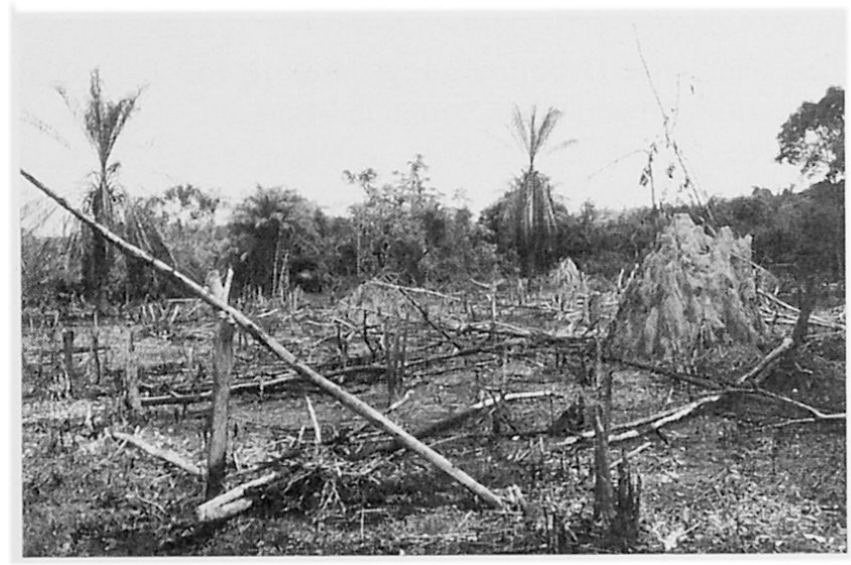

Abb. 2 Frische Rodung vor der Reissaat: Die Brandrodung hinterläßt ein mit Asche, Baumstümpfen und angekohlten dicken Ästen und Stämmen bedecktes Feld, welches so vor allzu starker Auswaschung und Erosion geschützt ist. Im Hintergrund stehen am Rand junger Buschbrachen angesengte Ölpalmen. Termitenhügel sind ein Kennzeichen für lateritische Böden.

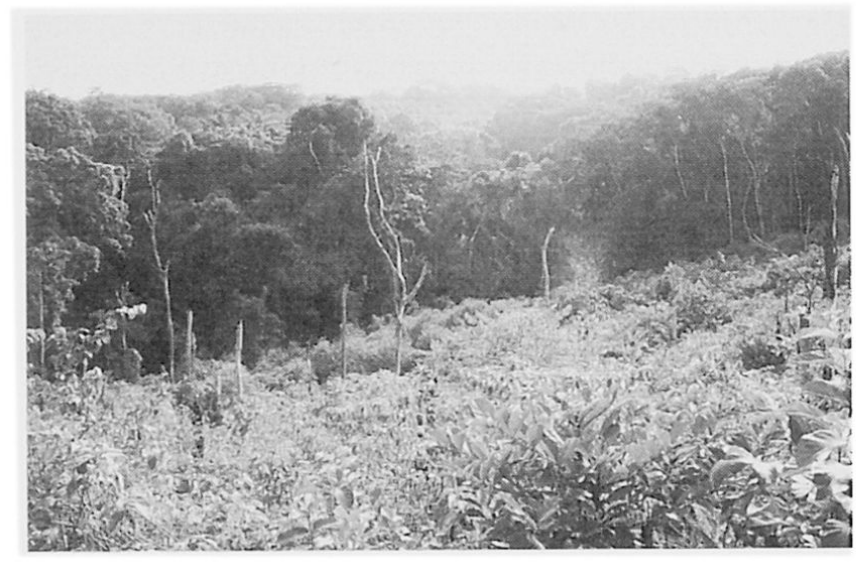

Abb. 3 Buschbrache im 1. Jahr. Inmitten des Feldes sind noch einige Maniokstauden zu erkennen. Im Hintergrund sind ältere Buschbrachen von altem Sekundärwald umgeben. 
bzw. Höhenklassen bilden das für die Feld-Wald-Wechselwirtschaft typische mosaikartige Flurbild. Ein Urwaldrelikt, das dem Poro-Geheimbund als Versammlungsort dient, ist direkt an der Lateritstraße nordwestlich des Dorfes zu erkennen. Gut die Hälfte der liberianischen Siedlungen sind solche Dörfer mit 100 bis 300 Einwohnern.

Das Dorf Zimgbo Town zählte 198039 Häuser, 36 Kochhütten und über 250 Einwohner des Gola-Stammes (Die 80000 Golas zählen zur westatlantischen Mel-Sprachgruppe). Das heutige Dorf geht auf 1893 zurück. Die Bevölkerungsdichte beträgt etwa $40 \mathrm{Ew} . / \mathrm{km}^{2}$, was zu hoch ist für dieses Agrarsystem.

Da das Dorf verkehrsmäßig abseits liegt (80 Straßenkilometer von Monrovia entfernt), ist die traditionelle Lebensweise einigermaßen erhalten geblieben. Die Großfamilie löst sich allerdings mehr und mehr auf, bereits herrscht die Einzelfamilie vor. Schon vor dem Bürgerkrieg war ein stärkerer Kulturwandel festzustellen. Wie viele Länder Afrikas hat auch Liberia einen hohen Anteil an junger Bevölkerung. Rund $43 \%$ der Einwohner sind jünger als 15 Jahre, was auch für Zimgbo Town zutreffen mag und bei einem Bevölkerungswachstum von 3,1\% nicht erstaunt. Eine bescheidene medizinische Versorgung hat die ehemals sehr hohe Kinder- und Säuglingssterblichkeit verringert. Die traditionelle Erziehung wird derzeit durch Staats- oder Missionsschulen von einem neuen Wertsystem überlagert. Es gibt jedoch immer noch 61\% Analphabeten. Seit Ende der 70er Jahre gibt es im Dorf eine Schule und eine Methodistenkirche.

\section{b) Produktionsmethoden und jahreszeitlicher Ablauf}

1. Zu Beginn der Trockenzeit (Dezember/Januar) wählt jede Familie ein Waldstück aus, in dem die Reispflanzung angelegt werden soll. In der Auswahl ist jedermann frei, denn nach altem Gewohnheitsrecht gehört der Boden der Stammesgemeinschaft. Jedermann hat das Recht, jährlich ein Stück Wald zu roden und zu nutzen. Das Nutzungsrecht auf dieser Rodung bleibt dann so lange erhalten, als die Arbeit des Pflanzers in der Rodung ersichtlich ist. Das arbeitsintensive Roden für das etwa 2 ha große Landstück, das von den Männern durchgeführt wird, nimmt mehrere Wochen in Anspruch. Dabei werden die kleineren Bäume und das Gestrüpp mit dem Buschmesser abgeschlagen, größere Bäume schlägt man mit der Axt an, damit sie absterben, oder man läßt sie stehen als Schattenspender. Das Abbrennen der inzwischen dürr gewordenen Pflanzen geschieht vor dem Einsetzen der eigentlichen Regenzeit. Zurück bleibt ein mit Holzasche, Baumstümpfen und angekohlten dicken Ästen und Stämmen bedecktes Feld, das die Gola absichtlich nicht weiter säubern, um die Erosion und Auswaschung infolge der vielen heftig niederprasselnden Gewitterregen abzuschwächen. Dem Brand kommt eine wichtige Funktion zu. Neben der Vernichtung von Unkraut, die dem Landwirt die mühsame Bodenbearbeitung erspart, fallen durch das Brennen große Mengen Aschendünger an, die die mangelhafte Bodenfruchtbarkeit zumindest temporär kompensieren. In jeder Reispflanzung wird eine kleine Hütte errichtet. Sie dient als Küche, während der Mittagshitze als Aufenthaltsraum und während der Ernte als Lagerplatz.

2. Die nun folgende Feldarbeit obliegt den Frauen. Nach dem Einsetzen der Regenzeit im April säen sie den Reis aus. Dazwischen pflanzen sie etwas Bohnen, Auberginen, Cayennepfeffer (Chillies) und andere Gewürze. Bei der Reishütte setzen sie Maniok, Mehlbananen, Ananas und manchmal Zuckerrohr. Die Reispflanzung ist eine Mischkultur; wo fast alles wächst, was man zum Essen braucht, und wo auch die wild wachsende Ölpalme häufig vorkommt. Die Auswahl und die Reihenfolge der Feldfrüchte erfolgen nach einem wohldurchdachten Anbauplan. Sobald der Reis zu keimen beginnt, ebenso wenn er ausreift, müssen die Kinder tagelang die Webervögel, die Spatzen Afrikas, verscheuchen, denn sonst richten diese großen Schaden an. Mehrmals jäten die Frauen das Unkraut, das in diesem Klima besonders schnell heranwächst.

Hackbau ist eine andere Bezeichnung für die afrikanische Landwirtschaft. Zum Säen, Pflanzen und Jäten benützen die Frauen kurzstielige Hacken. Ein Pflug könnte in diesen Rodungen, wo Baumstrünke und Wurzelwerk nicht entfernt sind, gar nicht eingesetzt werden. Zudem gibt es auch keine Zugtiere.

3. Von Ende Oktober bis Anfang Dezember hinweg erstreckt sich die Reisernte, denn die verschiedenen Reissorten haben unterschiedlich lange Wachstumszeiten. Die reifen Rispen werden mit einem Erntemesser abgeschnitten und bündelweise auf einem Bambusrost unter dem Dach der Hütte gelagert. Ein Küchenfeuer trocknet den Reis. Danach drischt man ihn, füllt ihn in große Körbe und bringt ihn in die Küche ins Dorf.

\section{c) Anbauprodukte und Ernteerträge, Tierhaltung, Jagd und Fischfang}

Das wichtigste Grundnahrungsmittel ist der Reis. Man verwendet verschiedene Sorten des Bergreises, der nicht bewässert werden muß. Täglich stehen etwa $350 \mathrm{~g}$ Reis pro Kopf zur Verfügung.

Die nicht bewässerten Bergreissorten ergeben sehr kleine Erträge: 540 bis $900 \mathrm{~kg} / \mathrm{ha}$. (Beim bewässerten Reisanbau sind die Erträge 5- bis $6 \mathrm{mal}$ größer, und dazu sind erst noch 2 Ernten pro Jahr möglich.)

Neben dem Reis ist der Maniok das zweitwichtigste Nahrungsmittel. Aus Südamerika stammend, hat er in Westafrika dieselbe Bedeutung erlangt wie in Europa die Kartoffel. Die stärkehaltigen Wurzelknollen des Maniokstrauches können ab dem zehnten Monat geerntet werden; sie können aber auch bis zu zwei Jahre im Boden belassen werden. So gibt es keine Lagerungsprobleme. Da der Maniok eine der ertragreichsten Nährpflanzen der Welt ist, sind nur kleine Anbauflächen nötig. Auf guten Böden kann man mit $7 \mathrm{t}$ pro Hektar rechnen. Viele Familien legen sich eine kleine Maniokpflanzung in Dorfnähe 
an oder bepflanzen einen kleinen Teil des Reisfeldes im zweiten Anbaujahr, wenn die Bodenfruchtbarkeit nachgelassen hat, mit Maniok. Obwohl er noch auf den minderwertigsten Böden wächst, sind die Erträge auf allzu häufig genutztem Boden nicht mehr zufriedenstellend.

Die Ölpalme ist eine der wichtigsten ölliefernden Pflanzen, deren Öl sowohl aus dem Fruchtfleisch (Palmöl) als auch aus den Samen der Steinkerne (Palmkernöl) gewonnen wird. Bei den Gola wächst sie wild. Die Fruchtstände setzen sich aus über 2000 pflaumengroßen Steinfrüchten zusammen und können bis zu $50 \mathrm{~kg}$ Gewicht erreichen. Das faserige, hellorange Fruchtfleisch der Steinfrucht enthält 50-70\% Fett. Die Ölpalme gedeiht nur im feuchttropischen Klima. Die Früchte der Ölpalme bilden eine wichtige Ernährungs- und Erwerbsgrundlage der liberianischen Stammesbevölkerung.

Ums Dorf herum zieht sich ein schmaler Kranz von Fruchtbäumen mit Gärten. In diesem Fruchthain kommen Orangenbäume, Kokospalmen, auch Mango-, Brotfrucht-, Avocado-, Kola- und Guavabäume vor sowie Mehl- und Obstbananen, und im Gärtchen dahinter gedeihen Papayamelonen, Süßkartoffeln, Ingwer, Linsen und andere Gemüse.

In manchen Dörfern hält man einige Hühner, Schafe oder Ziegen. Großvieh kommt nirgends vor wegen der Tsetsefliege, die mit ihrem Stich die Naganaseuche verbreitet.

Tierhaltung, Jagd, Fischfang sind von geringer Bedeutung für die Gola, denn in diesem schon seit langer Zeit genutzten Gebiet gibt es nicht mehr viel Wild. Es ist jedoch nötig, die Reispflanzung mit Stecken und Palmwedeln einzuzäunen, um größere Nagetiere, wie z. B. die Rohrratte, von der Pflanzung fernzuhalten.

In allen Teilen des Landes sind Sumpfgebiete und periodisch überschwemmte Talauen vorhanden, die mit ihren Fischen und Krebsen einen wertvollen Beitrag zu der allgemein sehr eiweißarmen Ernährung zu liefern vermögen. Es gehört deshalb zum wöchentlichen Arbeitsrhythmus der Frauen und Mädchen, mit Rundnetz und Fischreuse zum Fischfang für die Familie oder auch für den Markt zu gehen.

\section{d) Anbauzyklus, Nutzungsdauer und Bracheperioden}

Der Gola weiß aus Erfahrung, daß eine Reispflanzung nicht ein zweites Jahr mit Reis genutzt werden darf; bei gewaltigen Ertragsabfällen würde sich die Arbeit nicht lohnen. Deshalb gibt er die Pflanzung nach einjähriger Nutzung größtenteils auf und rodet ein neues Waldstück. Obwohl ein hohes und konstantes Angebot an Sonnenenergie und Wasser zur Verfügung steht, ist die Bodenfruchtbarkeit mangelhaft. Die Ursachen liegen vor allem in der starken Auswaschung der Bodennährstoffe durch die hohen Niederschläge, in der geringen Kationenaustauschkapazität der vorherrschenden zweischichtigen Tonmineralien und in der allgemein nur dünnen Humusauflage. Die von Eisen- und Aluminiumoxiden (Sesqui- oxiden, vor allem im A-Horizont) angereicherten Rotlehme (ferrallitische Böden) weisen zudem eine hohe Bodenazidität auf. Leider hält der Düngeeffekt der Brandrodung nur kurze Zeit an. Die bekannten kräftigen tropischen Regenschauer und die intensive Sonnenstrahlung sorgen für einen raschen Abbau der Asche und der dünnen Humusschicht. Deshalb werden die Pflanzungen nach ein- bis höchstens zweijähriger Nutzung der Verbuschung überlassen, so daß man jährlich neue Rodungen anlegen muß.

Buschbrachen: Schon im ersten Jahr nach dem Verlassen der Pflanzung wächst ein dichter Busch heran. Die alten Baumstrünke schlagen aus, neue Bäumchen wachsen schnell auf 2 m empor. Im Laufe von wenigen Jahren bildet die Buschbrache dank üppigem Wachstum der Pionierbäume ein Dickicht, welches schon nach 4 Jahren bis $7 \mathrm{~m}$ hoch ist. Bald entwickelt sich daraus junger Sekundärwald, welcher nach etwa 8 Jahren schon über $10 \mathrm{~m}$ Höhe erreicht. Nach 10-15 Jahren werden die Pionierbäume von den heranwachsenden Hochwaldbäumen allmählich verdrängt. Dieser ältere Sekundärwald ist 10-20 m hoch. Noch ältere Sekundärwälder gibt es hier nicht mehr.

Der nachwachsende Wald wird infolge starken Bevölkerungswachstums nach durchschnittlich 8 Jahren wieder gerodet, einzelne Buschwaldareale aber schon nach 3-5, andere erst nach 10 bis 15 Jahren. Nach 8 Jahren ist aber der einstige Stand der Bodenfruchtbarkeit noch nicht erreicht, das Gleichgewicht zwischen Anbau und Regeneration ist gestört. Die Erträge werden kleiner als früher, und dies erst recht bei noch kürzerer Brache.

Die gesamte Nutzfläche umfaßt pro Jahr 13\% der Dorffläche, wobei über $12 \%$ temporäre Anpflanzungen sind. Von diesen Feldern werden nur 15\% im zweiten Jahr mit Maniok bepflanzt. Durchschnittlich sind pro Jahr rund $10 \%$ der Dorffläche als frische Rodungen anzulegen.

Zusammenfassend ist festzustellen, daß die durchschnittliche Bracheperiode 8 Jahre dauert, wobei vom jährlich gerodeten Land rund 15\% zwei Jahre lang und $85 \%$ davon nur ein Jahr lang bewirtschaftet werden.

Über $40 \%$ des Areals werden von Buschbrachen eingenommen; das sind Buschdickichte im Alter von 1-5 Jahren, sie sind 2-7 m hoch. $38 \%$ sind mit Sekundärwald bewachsen, und zwar 23\% mit jungem, 7-10 m hohem Wald und $15 \%$ mit 10-20 m hohem, über 15 Jahre altem Wald. Der Rest verteilt sich auf Urwaldrelikte, Sümpfe (zusammen $4 \%$ ), auf Baumkulturen am Dorfrand und einige kleine Kautschukpflanzungen.

\section{e) Schlußfolgerungen}

Der Brandrodungsfeldbau ist eine höchst flächen- und arbeitsaufwendige Landwirtschaft, denn bei 8jähriger Brachedauer braucht das Dorf 9mal soviel Land, wie es in einem Jahr in Nutzung hat, und das jährliche Roden ist ein hoher Arbeitsaufwand, der nichts bringt. Zudem ist alles noch Handarbeit. Entsprechend gering ist die Trag- 
fähigkeit. Ein Familienbetrieb mit 6-7 Personen benötigt rund 18 ha Land, wovon jeweils nur 2 ha unter Kultur stehen. Bei einer Bevölkerungsdichte von $40 \mathrm{Ew} . / \mathrm{km}^{2}$ reicht dies gerade noch knapp. Das Bevölkerungswachstum führt zwangsläufig zu einer Ausdehnung der Anbaufläche und zu einer Verkürzung der Brachezeiten und damit $\mathrm{zu}$ einer weiteren Abnahme der Bodenfruchtbarkeit. Schon jetzt produzieren nicht alle Haushalte genügend Reis.

Immer mehr junge Männer versuchen ihr Glück in Monrovia, wo aber nur wenigen ein wirtschaftlicher und sozialer Aufstieg gelingt. Einige sind saisonal beschäftigt in der benachbarten Kautschukplantage, welche am nördlichen Dorfrand Anfang der sechziger Jahre errichtet wurde. Viele sind unterbeschäftigt, denn bei gemeinsamer Arbeit erfordert die Landwirtschaft pro erwachsene Person nur etwa 100 Arbeitstage. Weil auch wenig Überschüsse für den Markt produziert werden, ist das Einkommen entsprechend gering. Pro Kopf und Jahr stehen nicht mehr als etwa Fr. 200.- zur Verfügung.

In Liberia leben etwa 1,5 Millionen Einwohner (60-70\% der Bevölkerung) vom Brandrodungsfeldbau. Diese Hackbauern roden jährlich etwa $4500 \mathrm{~km}^{2}$ Busch und Wald (meist Sekundärwald). Bei durchschnittlich 10jähriger Brache beanspruchen sie rund $50000 \mathrm{~km}^{2}$ und damit gegen die Hälfte des Landes (gesamte Landfläche: $111369 \mathrm{~km}^{2}$ ).

Bei geringer Bevölkerungsdichte ist der Brandrodungsfeldbau ein ökologisch gut angepaßtes Landnutzungssystem. Beim gegenwärtigen Bevölkerungswachstum hat er aber keine Zukunft. Lösungen für eine produktivere Landwirtschaft wären dringend erforderlich, leider sind sie aber in Liberia kaum über das Versuchsstadium hinausgekommen.

Agrartechnische Lösungsmöglichkeiten wären Naßreisanbau auf alluvialen Schwemmlandböden, marktorientierte Baum- und Strauchkulturen in Verbindung mit Hausgärten, die vielfältige Kombinationsmöglichkeiten von Dauerkulturen mit einjährigen Nutzpflanzen zur Selbstversorgung anbieten. Wichtig ist dabei der waldartige Anbau (sogenannte Agroforstwirtschaft), bei welchem die Aschendüngung durch das ergiebigere Mulchen ersetzt wird, um den geschlossenen Nährstoffkreislauf beizubehalten. Die ausreichende Produktion von und Versorgung mit landwirtschaftlichen Produkten ist jedoch in erster Linie ein gesellschaftliches, wirtschaftliches und politisches Problem.

\section{Literatur}

DOPPLER, W. (1994): Landwirtschaftliche Betriebssysteme in den Tropen und Subtropen. In: Geograph. Rundschau 2/94, 65-71.

KAPPEL, R., und KORTE, W. (1993): Liberia. In: NOHLEN, D., und NUSCHELER, F: Handbuch der Dritten Welt, Band 4, 278-297, Bonn.

KOCH, P. (1970): Die Shifting Cultivation und ihre Luftbildauswertung (Diss.), Zürich.

KOCH, P. (1990): Gola und Dan - zwei Bauernvölker im tropischen Regenwald von Liberia. 2 Diaserien à 25 und 10 Dias mit Informationsmappe, Adligenswil (Eigenverlag).

MANSHARD, W. (1988): Entwicklungsprobleme in den Agrarräumen des tropischen Afrika, Darmstadt.

SCHOLZ, U. (1991): Überlegungen zum Agrarpotential und zur Tragfähigkeit tropischer Regenwaldgebiete. In: Tropischer Regenwald als Ökosystem, Gießener Beiträge zur Entwicklungsforschung Reihe I, Band 19, 41-53, Justus-LiebigUniversität, Gießen.

WEISCHET, W. (1980): Die ökologische Benachteiligung der Tropen, Stuttgart (2. Auflage).

ZIMMERMANN, G. (1992): Der tropische Regenwald in Kalimantan. In: Geograph. Rundschau 1/92, 40-47. 


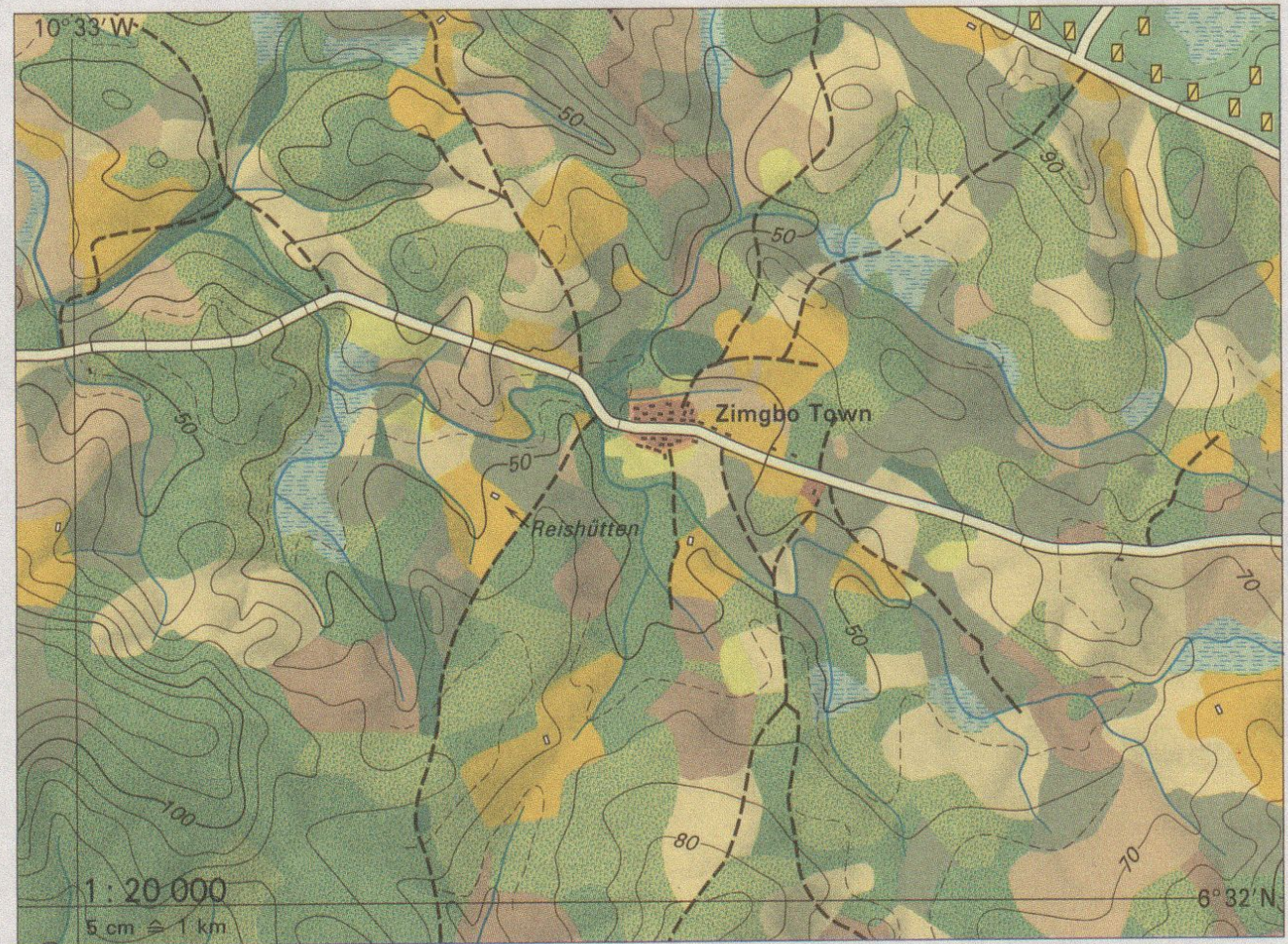

\begin{tabular}{|c|c|c|c|}
\hline \multirow{4}{*}{$\begin{array}{l}\text { Brandrodungsfeld- } \\
\text { bau in Liberia } \\
\text { Hauptpflanzung: Reis in Mischkultur } \\
\text { mit Maniok, Pfeffer u. a.. Waldbrache } \\
1 \text { Jahr Nutzung, } 8 \text { Jahre Waldb }\end{array}$} & \multirow{4}{*}{ 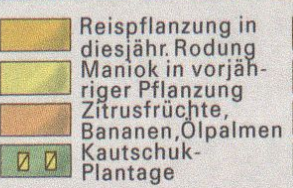 } & $\begin{array}{l}\text { Buschbrache } \\
\text { im 1. Jahr }(1-2 \mathrm{~m})\end{array}$ & \multirow{4}{*}{$\begin{array}{l}\text { junger Sekundär- } \\
\text { wald }(7-10 \mathrm{~m}) \\
\text { alterer Sekundär- } \\
\text { wald }(10-18 \mathrm{~m} \text { ) } \\
\text { Hochwald, Galerie- } \\
\text { wald (über } 18 \mathrm{~m}) \\
\text { Sumpf und Bambus }\end{array}$} \\
\hline & & im 2. Jahr $(2-3 \mathrm{~m})$ & \\
\hline & & im 3. Jahr $(3-4 \mathrm{~m})$ & \\
\hline & & $(4-7 \mathrm{~m})$ & \\
\hline
\end{tabular}

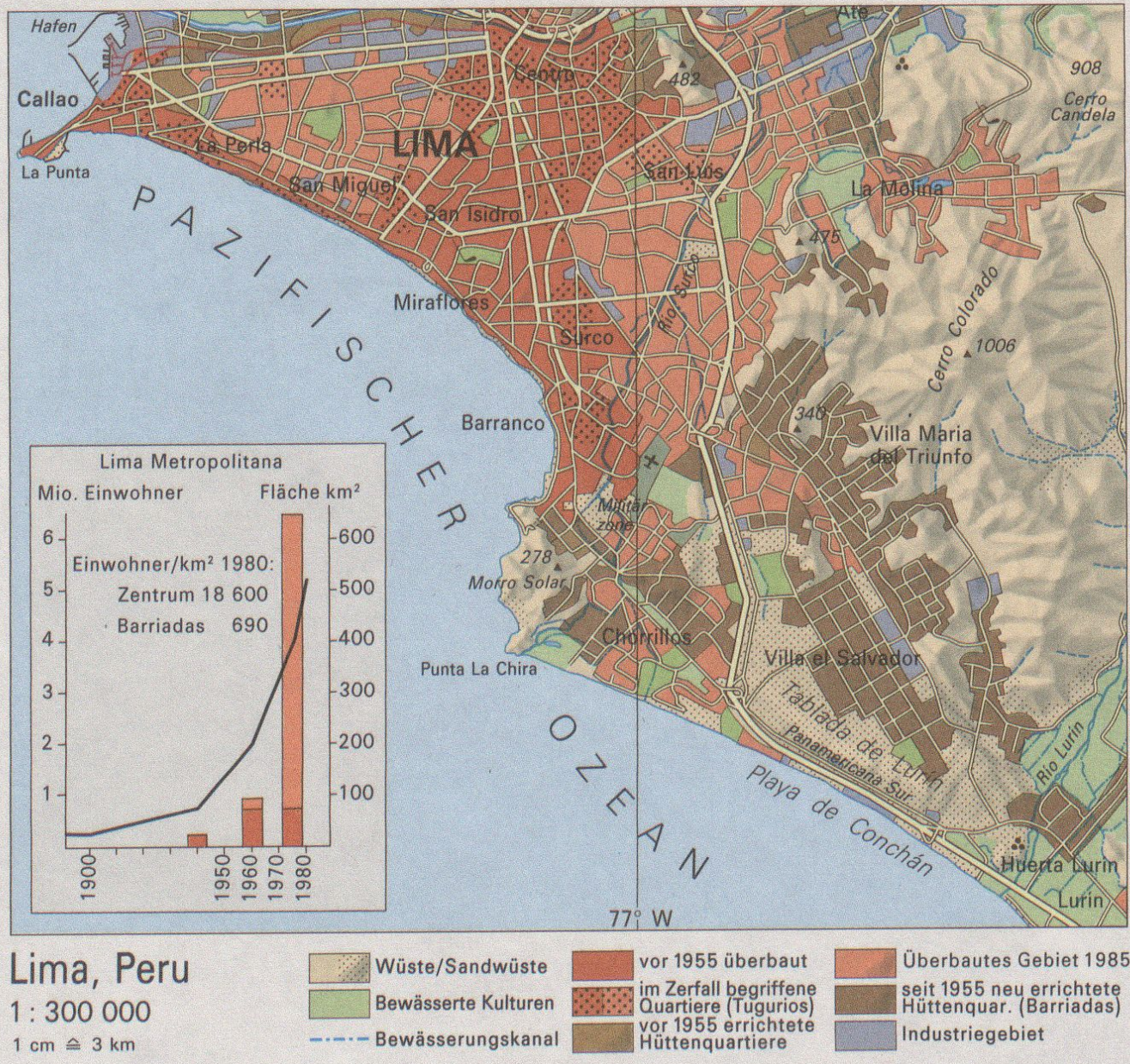





\section{Wirtschaftsformen \\ - Mediterrane Landwirtschaft Hirten und Viehzüchter Savannenpflanzer \\ - Waldlandpflanzer \\ - - Oasenbauern \\ - - Steppenjäger \\ ......... Südafrikanische Farmwirtschaft \\ - Pygmäen (Urwaldjäger)}

